\title{
Elzacher Konzept und Leistungskatalog der therapeutischen Pflege in der neurologischen Frührehabilitation (Phase B)
}

\section{Elzach Concept and Intervention Catalogue of Nursing Therapy in Postacute Neurological Rehabilitation within the German DRG System}

\author{
Autoren \\ J. Himaj ${ }^{1}$, E. Müller ${ }^{1}$, B. Fey ${ }^{1}$, S. Neumaier ${ }^{2}$, B. Waibel ${ }^{2}$, P. Dirschedl², C.-W. Wallesch ${ }^{1}$ \\ Institute \\ ${ }^{1} \mathrm{BDH}-$ Klinik Elzach \\ ${ }^{2}$ Medizinischer Dienst der Krankenversicherung Baden-Württemberg, Lahr
}

\section{Schlüsselwörter \\ - neurologische Frührehabilitation \\ - Pflegetherapie \\ - DRG-System \\ Key words \\ - neurological early rehabilitation \\ - nursing therapy \\ - DRG system}

Bibliografie DOI http://dx.doi.org/ 10.1055/s-0030-1263189 Rehabilitation 2011; 50: 94-102 (c) Georg Thieme Verlag KG Stuttgart · New York ISSN 0034-3536

Korrespondenzadresse Prof. Dr. Claus-W. Wallesch BDH-Klinik Elzach Am Tannwald 1-3 79215 Elzach claus.wallesch@neuroklinikelzach.de

\section{Zusammenfassung}

Die im DRG-System erforderliche Dokumentation pflegetherapeutischer Leistungen und die Begutachtung ihrer Erbringung durch den Medizinischen Dienst der Krankenversicherung (MDK) gestaltet sich schwierig. Vor allem eine eindeutige Trennung von Grund- und therapeutischer Pflege ist aus pflegewissenschaftlicher Sicht problematisch. Um Reibungsverluste und Schnittstellenprobleme zu minimieren, haben sich Leistungserbringer und MDK zunächst für Baden-Württemberg auf einen Leistungskatalog verständigt. Elzacher Konzept und Leistungskatalog beschreiben die therapeutische Pflege und sind durch die Eingrenzung der Leistungen sowohl für den Leistungserbringer als auch für die Kostenträger bzw. den MDK überschaubar und transparent.

\section{Einleitung}

Die neurologisch-neurochirurgische Frührehabilitation der Phase B nach Bundesarbeitsgemeinschaft für Rehabilitation (BAR) [1] ist in den meisten Bundesländern leistungsrechtlich der Krankenhausbehandlung zugeordnet. Sie unterliegt somit dem System der Diagnosis Related Groups (DRG), sofern der Leistungserbringer nicht den Status einer „besonderen Einrichtung“ hat.

Das deutsche DRG-System bildet spezielle nichtoperative Leistungskomplexe wie z.B. die neurologische Komplexbehandlung des Schlaganfalls auf der Stroke Unit (Operationen- und Prozedurenschlüssel - OPS 8-981), die Komplexbehandlung bei Besiedelung oder Infektion mit multiresistenten Erregern (OPS 8-987) und eben auch die neurologisch-neurochirurgische Frührehabilitation (OPS 8-552) über einen Katalog von Mindestmerkmalen ab, die die Qualität der Leistungserbringung sichern und diese auch einer Über-

\section{Abstract \\ $\nabla$}

The German DRG system defines specialized treatments through a set of minimal requirements, the documentation of which and its assessment by external specialists of the medical service of the statutory health insurance causing frictions between rehabilitation hospitals and the medical service. It is especially problematic to make a clear distinction between nursing care and treatment. To reduce frictions and interface problems, a catalogue of acts of therapy performed by nurses was agreed for neurological early rehabilitation in Baden-Württemberg. This Elzach concept and catalogue describe therapeutic nursing in neurological rehabilitation and increase transparency and accountability both for providers and cost carriers. nahmen der Grundpflege als auch der therapeutischen Pflege beinhalten. Es ist offen, ob Kalkulationen auf der Grundlage der OPS-Gruppe 9-20 zu schweregradpartitionierten DRGs führen oder in diese eingehen oder ob sie Zusatzentgelte begründen werden. Der Erfassung der hochaufwendigen Pflege liegt ein grundsätzlich anderer Ansatz als den bereits bestehenden diagnoseabhängigen Komplexbehandlungen zugrunde; prinzipiell kann - von Kurzliegern abgesehen - jeder Patient in jeder DRG hochaufwendige Pflege im Sinne der OPS 9-20 benötigen. Zu dokumentieren 
sind sowohl die Gründe für die Notwendigkeit hochaufwendiger Pflege als auch die durchgeführten Maßnahmen.

Für alle Maßnahmen der OPS-Gruppe 8-55 (Frührehabilitative Komplexbehandlung) gilt, dass ein Kode aus diesem Bereich nur solange verwendet werden darf, wie akutstationärer Behandlungsbedarf besteht. Darüber hinaus gelten für die neurologischneurochirurgische Frührehabilitation nach OPS 8-552 folgende Mindestmerkmale:

1. Frührehateam unter Leitung eines Facharztes für Neurologie, Neurochirurgie ..., der über eine mindestens 3-jährige Erfahrung in der neurologisch-neurochirurgischen Frührehabilitation verfügt. Im Frührehateam muss der neurologische oder neurochirurgische Sachverstand kontinuierlich eingebunden sein.

2. Standardisiertes Frührehabilitations-Assessment zur Erfassung und Wertung der funktionellen Defizite in mindestens 5 Bereichen (Bewusstseinslage, Kommunikation, Kognition, Mobilität, Selbsthilfefähigkeit, Verhalten, Emotion) zu Beginn der Behandlung. Der Patient hat einen FrührehabilitationBarthel-Index nach Schönle bis maximal 30 Punkte zu Beginn der Behandlung.

3. Wöchentliche Teambesprechung mit wochenbezogener Dokumentation bisheriger Behandlungsergebnisse und weiterer Behandlungsziele.

4. Aktivierend-therapeutische Pflege durch besonders geschultes Pflegepersonal auf dem Gebiet der neurologischneurochirurgischen Frührehabilitation.

5. Vorhandensein und Einsatz von folgenden Therapiebereichen: Physiotherapie/Krankengymnastik, Physikalische Therapie, Ergotherapie, Neuropsychologie, Logopädie/fazioorale Therapie und/oder therapeutische Pflege (Waschtraining, Anziehtraining, Esstraining, Kontinenztraining, Orientierungstraining, Schlucktraining, Tracheostomamanagement, isolierungspflichtige Maßnahmen u.a.) in patientenbezogen unterschiedlichen Kombinationen von mindestens 300 min täglich (bei simultanem Einsatz von 2 oder mehr Mitarbeitern dürfen die Mitarbeiterminuten aufsummiert werden) im Durchschnitt der Behandlungsdauer der Frührehabilitation.

Die Problematik, wie die Erfüllung der Mindestmerkmale dokumentiert werden kann, und Argumentationslinien in der Auseinandersetzung mit dem MDK wurden bereits an anderer Stelle beschrieben [2]. Hauptgegenstand der Kritik des MDK sind Inhalte und Dokumentation der aktivierend-therapeutischen Pflege und ihre Abgrenzung von Leistungen der Grundpflege [2].

Zur Kodierung von Prozeduren im Rahmen des OPS führt die Deutsche Krankenhausgesellschaft [3] aus: „Die schlüssige Übereinstimmung von klinischer Dokumentation und der Codierung von Diagnosen und Prozeduren unter Beachtung der deutschen Codierrichtlinien ist unabdingbar, um Auseinandersetzungen mit den Kostenträgern bzw. Entgeltkürzungen nach MDK-Prüfverfahren zu vermeiden“ (S. 28). „Zur Sicherstellung einer schlüssigen Übereinstimmung der klinischen Dokumentation mit der Codierung der Prozeduren unter Beachtung der Codierrichtlinien ist insbesondere Folgendes zu berücksichtigen: ... Belege für die Erfüllung der im OPS geforderten Mindestmerkmale bei der Abrechnung von sogenannten Komplexcodes" (S. 30/31).

Hier wurde seitens des MDK wiederholt ausgeführt, dass die Gewährleistung des in der Definition der Mindestmerkmale hervorgehobenen Trainingsaspekts sowie die Abgrenzung von Leistungen der Grundpflege eine detaillierte einzelleistungsbezogene Dokumentation erforderten, wenn die gesamte (gemes- sene) Zeit der Leistungserbringung als Therapiezeit gewertet werden solle [2]. Alternativ kann dem MDK-Gutachter ein bestimmter Leistungsumfang plausibel gemacht werden, wenn ein Katalog von Leistungen der aktivierend-therapeutischen Pflege, denen Plausibilitätszeiten zugeordnet sind, herangezogen wird. Ein solcher „Katalog der therapeutischen Pflege (KtP) in der neurologisch-neurochirurgischen Frührehabilitation (Phase B)“ wurde von den Arbeitskreisen Neurologischer Kliniken in Bayern und Thüringen in Zusammenarbeit mit dem MDK Bayern entwickelt [4].

Im Dialog mit dem MDK Baden-Württemberg wurde deutlich, dass zum einen eine detailliertere Definition der Inhalte der einzelnen pflegetherapeutischen Leistungen wünschenswert ist, als sie im bayerisch-thüringischen Katalog gegeben wird, wodurch die Abgrenzung von pflegetherapeutischen Leistungen von reinen Grundpflegeverrichtungen geschärft werden soll. Dies erscheint auch deshalb sinnvoll, weil das Thema der aktivierend-therapeutischen Pflege bisher pflegewissenschaftlich ungenügend bearbeitet worden ist. Die Grundlage für die in die Verhandlung eingebrachten therapeutischen Leistungen und Plausibilitätszeiten bildeten die Systematik der Leistungen des bayerisch-thüringischen Kataloges [4], ein bislang in der BDHKlinik Elzach verwendeter Katalog von Leistungen der aktivierend-therapeutischen Pflege in der neurologischen Frührehabilitation, der nicht mit Plausibilitätszeiten hinterlegt war, sondern eine Zeiterfassung beinhaltete, und in der BDH-Klinik Elzach erhobene Durchschnittszeiten der Leistungserbringung je Position des Kataloges. Zum anderen erschien im bayerischthüringischen Katalog die Unterscheidung in aktivierende vollkompensatorische, aktivierende teilkompensatorische und anleitend-begleitende Pflegetherapie verzichtbar. Oft liegen nicht klar zuordenbare Mischformen vor, sodass keine reliablere Zeitermittlung durch die Verwendung der Kategorien erwartet werden darf.

In anderem Kontext (nämlich der ambulanten Pflege) führt eine „Grundsatzstellungnahme Pflegeprozess und Dokumentation“ des Medizinischen Dienstes der Spitzenverbände [5] aus: „Die Durchführung der Maßnahmen wird mit Handzeichen und Uhrzeit ... auf dem Durchführungskontrollblatt durch die durchführenden Mitarbeiter dokumentiert" (S. 31).

Es wurde mit dem MDK vereinbart, dass dies auch für die aktivierend-therapeutische Pflege sowie für weitere Pflegemaßnahmen in der Frührehabilitation zu gelten habe, und es wurde ein entsprechendes Dokumentationsblatt entwickelt, auf dem schichtweise die entsprechenden Handzeichen zu hinterlegen sind. Perspektivisch erscheint eine elektronische Dokumentation und Signatur der Leistungserbringung möglich und wünschenswert, wie sie von Wendland [6] für die Erfassung nach KtP [4] und KTL (Klassifikation therapeutischer Leistungen in der medizinischen Rehabilitation) vorgestellt wurde. Es müsste dazu allerdings eine Vereinbarung über die Überprüfbarkeit der elektronischen Signatur getroffen werden.

Um eine einfache und praktisch handhabbare Leistungsdokumentation zu ermöglichen, erfolgte eine Einigung auf 30 pflegetherapeutische Leistungen, für die Plausibilitätszeiten vereinbart wurden (Anhang 1). Bei der Festlegung der Plausibilitätszeiten wurden durchschnittliche Anteile grundpflegerischer Leistungen diskutiert und bei der Festlegung der Plausibilitätszeiten pragmatisch berücksichtigt. Der hier vorgestellte Katalog löst das Problem der Abgrenzung der Grund- von der therapeutischen Pflege nicht, sondern ist ein pragmatischer Ansatz zur Dokumentation der Erbringung der OPS 8-552. Die Definition 
und pflegetheoretische Begründung der aktivierend-therapeutischen Pflege stellt eine wichtige Herausforderung an die Pflegewissenschaft dar, deren Bearbeitung der BDH - Bundesverband Rehabilitation - mit der Ausschreibung eines Promotionsstipendiums Pflegewissenschaften unterstützt. Im Rahmen dieser Forschung soll der hier vorgestellte Katalog empirisch evaluiert und pflegetheoretisch analysiert werden.

Der MDK hat sich damit einverstanden erklärt, dass die Dokumentation von Inhalten, Behandlungsergebnissen und Zielen der Pflegetherapie wie bei den anderen Therapien (Physiotherapie, Ergotherapie, Logopädie, Neuropsychologie) wochenbezogen erfolgt, wie dies die Legende zur OPS 8-552 (s. oben) auch nahelegt. Eine Reihe weiterer Vereinbarungen wurde in den Erläuterungen zum Katalog niedergelegt.

Wir glauben, dass das erzielte Ergebnis eine transparente Dokumentation der Pflegetherapie erlaubt, den Zeitaufwand aufseiten der Leistungserbringer in vertretbaren Grenzen hält und dem MDK die Prüfung erleichtert, ob die Mindestmerkmale hinsichtlich der Leistungen der aktivierend-therapeutischen Pflege erfüllt wurden. Die Publikation des Katalogs soll anderen Leistungserbringern die Möglichkeit geben, diesen anzuwenden. Aufgrund der konkreten Auswirkungen auf Dokumentation und Prüfverfahren empfehlen die Autoren, bei einer geplanten Anwendung des Katalogs mit dem jeweils zuständigen MDK die Akzeptanz des Katalogs zu klären.

\section{Kernbotschaft}

Um die Erbringung, Dokumentation und Begutachtung von Komplexbehandlungen im OPS unstrittig zu stellen, sind Kooperationen zwischen Leistungserbringer und MDK hilfreich.

\section{Literatur}

1 Bundesarbeitsgemeinschaft für Rehabilitation. Empfehlungen zur Neurologischen Rehabilitation von Patienten mit schweren und schwersten Hirnschädigungen in den Phasen B und C. Frankfurt a. M.: BAR; 1999

2 Wallesch $C-W$. Frührehabilitation und OPS 8-552. Akt Neurol 2009; 36: 93-97

3 Deutsche Krankenhausgesellschaft. Die Dokumentation der Krankenhausbehandlung - Hinweise zur Durchführung, Archivierung und zum Datenschutz. 3., geänd. Aufl. Düsseldorf: Deutsche Krankenhaus-Verlagsgesellschaft; 2007

4 Katalog der therapeutischen Pflege (KtP) in der neurologisch-neurochirurgischen Frührehabilitation (Phase B). 2007, verfügbar unter: www. enzensberg.de/index.shtml?ktp

5 Bruckner U, Ziegler G, Theis S, Jodes-Laßner U, Köhler C, Reus U, Uhl A, Veit-Zenz A. Grundsatzstellungnahme Pflegeprozess und Dokumentation. Essen: Medizinischer Dienst der Spitzenverbände der Krankenkassen; 2005

6 Wendland G. Kostenträger fordern Leistungstransparenz in der Pflege. Die Schwester Der Pfleger 2010; 49: 175-178 


\section{Anhang 1}

\section{Elzacher Konzept der therapeutischen Pflege $\nabla$}

\section{Erläuterungen}

Interdisziplinäre Patientenbesprechungen mit wochenbezogener Dokumentation bisheriger Behandlungsergebnisse und der Formulierung weiterer Behandlungsziele finden einmal wöchentlich statt. Dabei werden aus fachspezifischen Rehabilitationszielen fachübergreifende interdisziplinäre Ziele ausgewählt.

Die Koordination, Formulierung und kontinuierliche Anpassung konkreter interdisziplinärer Rehabilitationsziele, deren Dokumentation und die Überprüfung des Rehabilitationsverlaufs anhand dieser Ziele sind die Hauptaufgaben der interdisziplinären Besprechung. Die formulierten interdisziplinären Rehabilitationsziele gehen in den Arbeitsauftrag aller Beteiligten ein. Ihre Erarbeitung ist ein notwendiger Bestandteil der Rehabilitationsund Therapieplanung. Die Einrichtung verpflichtet sich, fortlaufend entsprechende qualifizierende Maßnahmen durchzuführen, das Besprechungsformat wird laufend evaluiert und verbessert.

Die Pflege ist Bestandteil des interdisziplinären Teams, ihre pflegetherapeutischen Leistungen erbringt sie durch besonders geschultes Personal.

Beim Lagern und beim Transfer wird bei simultanem Einsatz von 2 Mitarbeitern die Leistung doppelt angesetzt und durch Handzeichen dokumentiert. Die Notwendigkeit des Einsatzes zweier Mitarbeiter geht aus der Beschreibung des Zustandsbildes im Rahmen der Teambesprechung oder dem wochenbezogenen, schriftlichen Bericht des Pflegepersonals hervor.

Bei einem liegenden transurethralen Katheter wird kein Kontinenztraining angesetzt.

Abweichungen von Plausibilitätszeiten nach oben müssen zusätzlich nachvollziehbar im Pflegebericht oder auf einem Zusatzblatt dokumentiert werden.

Es ist keine exakte zeitliche Zuordnung der Leistungen notwendig. Die schichtbezogene Dokumentation (Früh-, Spät-, Nachtdienst) erfüllt die Anforderungen. Die jeweilige Leistung ist vom erbringenden Mitarbeiter durch Handzeichen zu dokumentieren.

Der Katalog beinhaltet Module, die miteinander kombiniert erbracht werden können. Die Abkürzung für Maßnahmen der therapeutischen Pflege lautet TP.

Der Zeitbedarf für die Teamkonferenz wird nicht in Ansatz gebracht. Pauschal werden tgl. 15 min Dokumentationszeit für die therapeutische Pflege angesetzt. Es ist selbstverständlich, dass nur Leistungen dokumentiert werden, die bezüglich Inhalt und Umfang den hinterlegten Definitionen entsprechend erbracht worden sind.

Der Verlauf der therapeutischen Pflege ist mit einem wochenbezogenen, schriftlichen Bericht mit Angaben zur Ausgangssituation und zu den durchgeführten Maßnahmen sowie den erreichten Fortschritten zu dokumentieren.

Die in Klammern gesetzten Teilverrichtungen sind gegebenenfalls in den Unterpunkten der Leistungsbeschreibung enthalten und lösen allein keine Hauptleistungen aus.

Eine Überarbeitung dieses Katalogs wird in regelmäßigen Abständen durchgeführt. Alle Änderungen erfolgen in Absprache mit dem MDK Baden-Württemberg.

\section{A) Grundsätzliches}

Patienten in der neurologischen Frührehabilitation (Phase B), z.B. im Wachkoma, mit schwersten Wahrnehmungsbeeinträchtigungen und/oder ausgeprägten Paresen/Plegien weisen komplexe Beeinträchtigungsmuster im kognitiven, emotionalen, sensorischen, kommunikativen und motorischen Bereich auf. Diese beeinflussen sich gegenseitig und können die daraus erwachsenden Fähigkeitsstörungen verstärken. Die Maßnahmen der therapeutischen Pflege sind darauf ausgerichtet, den Patienten im Hinblick auf Vigilanz, Kommunikation, bewusste Wahrnehmung und absichtsvolle Handlungen zu stimulieren, ungerichtete und unspezifische Unruhe zu mindern, seine eigenen Aktivitäten zu erkennen, zu unterstützen und auszubauen. Grundsätzlich wird der Patient in alle Verrichtungen aktiv einbezogen, wobei sich Art und Umfang nach den jeweiligen Ressourcen/Fähigkeiten sowie den Beeinträchtigungen richten. Selbsthilfetraining zur Anbahnung der Selbstpflegefähigkeit findet auch durch die therapeutische Gestaltung von Alltagssituationen (Körperpflege, Anziehen, Nahrungsaufnahme, Mobilisation usw.) statt.

Bei jeder pflegerischen Verrichtung wird mit dem Patienten verbal/nonverbal, mimisch/gestisch und taktil Kontakt aufgenommen. Bei Patienten im Wachkoma bzw. frühen Remissionsphasen erfolgt dies immer mit einer Initialberührung, begleitet von einer kurzen verbalen Begrüßung. Vor und bei jeder Verrichtung wird beobachtet, ob der Patient den Kontakt erwidern kann; es wird beobachtet, inwieweit er während der Handlung aufmerksam ist. Willentliche Reaktionen des Patienten werden einbezogen; wenn sie adäquat zur vorgenommenen Handlung sind, werden sie gefördert und ausgebaut. Alle Handlungen, bei denen zunächst keine Unterstützung durch den Patienten erfolgt, werden in Teilsequenzen „geführt“ durchgeführt. Die Prinzipien der verbalen, taktilen Vorbereitung und Stimulation sowie das „Führen“ dienen der Verbesserung der Wahrnehmung und damit auch der kognitiven Stimulation.

Generell gelten neurophysiologisch begründete Konzepte wie das Bobathkonzept, die Konzepte der Basalen Stimulation und der Kinästhetik als feste Bestandteile der therapeutischen Pflege. Förderangebote aus diesen Konzepten werden in der therapeutischen Pflege miteinander kombiniert und den individuellen Bedürfnissen des Patienten angepasst.

\section{B) Einzelmaßnahmen (die o.g. grundsätzlichen Prinzipien haben bei jeder Einzelmaßnahme Gültigkeit) \\ Training der Körperpflege}

Alle Maßnahmen zur Körperpflege stellen gleichzeitig Wahrnehmungsangebote an den Patienten dar. Der über die verschiedenen Sinneskanäle gegebene Input dient der Förderung der bewussten Wahrnehmung und Anregung der Eigenaktivität hin zu absichtsvollen Bewegungen.

Sowohl bei erhöhtem Tonus als auch bei schwerer vegetativer Dysregulation werden neurophysiologisch begründete Verfahren zur Tonussenkung angewandt, um eine Regulationsfähigkeit anzubahnen.

Verrichtungen können unter Handführung des Patienten durch den Pflegenden erfolgen. Der Betroffene erhält hierdurch eine basale Rückmeldung, die es ihm ermöglicht, sich an Altes, Erlerntes zu erinnern und auf diese Speicherung zurückzugreifen. Dies gilt es zu erkennen und zu unterstützen.

Beginnende sensorische/motorische Fähigkeiten des Patienten werden einbezogen. Bei schwer kognitiv beeinträchtigten 
Patienten sind es zunächst einzelne kurze Handlungssequenzen, die schrittweise ausgebaut werden. Der Patient wird dabei unterstützt, seine mehr betroffene Seite wieder wahrzunehmen (Hemineglect, Hemianopsie) und seine bewegungseingeschränkten Extremitäten (infolge Paresen usw.) zu benutzen.

Er erhält Schulung und Hilfe beim Umgang mit geeigneten Hilfsmitteln.

Bei Störungen in der Handlungsplanung, der Raumwahrnehmung und des korrekten räumlichen Handelns können im Setting der Körperpflege Struktur, zielgerichtetes Handeln und die räumliche Wahrnehmung trainiert werden.

\section{Anziehtraining}

Aktivierung des Patienten durch Führung bei zunächst einfachen Verrichtungen wie z.B. Einbringen einer Hand in ein Ärmelloch. Dann Aufbau komplexerer Handlungsabläufe wie z.B. T-Shirt anziehen; erweiterter Einsatz und Ausbau der Ressourcen des Patienten.

\section{Mobilisation}

(Langsitz oder Schneidersitz im Bett, Sitzen an der Bettkante, Pilotsitz): Förderung der Vigilanz und der Aufmerksamkeit. Schulung der Körperwahrnehmung durch veränderten vestibulären und propriozeptiven Input. Fördert die Erfassung der Umgebung über die eigene Wahrnehmung hinaus (Fernsinn: Auge, Nase, Gehör). Fördert die Aktivität von Nacken- und Rumpfmuskeln, erlaubt eine Tonusmodulation, fördert den willkürlichen Einsatz insbesondere der oberen Extremitäten. Schlucken und Abhusten werden erleichtert. Die Wahl der Mobilisation richtet sich nach der Belastbarkeit sowie insbesondere nach der Vigilanz und der sich durch die Mobilisation ergebenden Tonussituation. Diese erfordert ggf. eine Anpassung der Maßnahmen.

\section{Stand}

Bei Phase B-Patienten meist überwiegend passiv, d. h. mit 2 Therapeuten, ggf. im Standing oder Stehbett. Stabilisierung der Kniegelenke, des Beckens, ggf. auch Hilfe bei Rumpfaufrichtung und Kopfkontrolle. Förderung der Wahrnehmung des Untergrundes, der Aufrichtung des Rumpfes. Ziel ist dabei die Stärkung der gegen die Schwerkraft gerichteten Muskulatur, eine Tonusmodulation, die Anbahnung von Gleichgewichtsreaktionen und die Stimulation des Kreislaufs. Hierdurch kommt es zu einer Steigerung der Vigilanz, der bewussten Körperwahrnehmung und der Aktivierung des willentlichen Einsatzes der Stützund Haltemotorik.

\section{Lagewechsel und Positionierung}

Der Lagewechsel aktiviert den Patienten über die aktive und passive Mobilisierung, über die Unterstützung möglicher Eigenaktivität sowie durch die Modulierung und Reduzierung eines unerwünscht hohen Muskeltonus. Dies ist ein wesentlicher Schritt, um willkürliche Bewegungen und sinnvolle unwillkürliche Unterstützungsmotorik zu ermöglichen.

Transfer (Übersetzen vom Bett in den Rollstuhl und zurück, ggf. Stuhl oder WC)

Förderung von Wahrnehmung und Motorik wie bei der Mobilisation. Zudem Aktivierung der Wahrnehmung des Untergrundes im Bereich von Gesäß und Fußsohlen, der Gelenkstellung sowie der Muskelspannung. Aktivierung der Gleichgewichtsreaktionen. Verbesserung der Wahrnehmung für Bewegung im Raum. Förderung der Kreislaufregulation und -stabilität. Die Techniken werden den Fähigkeiten des Patienten entsprechend ausgewählt.

Beispiele:

a) Tiefer Transfer passiv: wenn Patient nicht mithelfen kann (Coma vigile, Tetraplegie usw.)

b) Tiefer Transfer mit aktiver Mithilfe des Patienten: Patient mit Hemisymptomatik, der mit der weniger betroffenen Seite mithelfen kann.

c) Transfer über Stand: Patient kann stehen, dabei wird jedoch ein bestimmtes Ziel verfolgt, z. B. Verbesserung der Rumpfstabilität, Tonusregulation, Schulung der Standbein- und Spielbeinphase usw.

\section{Dysphagietherapie}

Diese erfolgt in Kooperation mit den Mitarbeitern der Ergotherapie und Sprachtherapie. In der Frühphase steht die Erarbeitung des Haltungshintergrundes und der Kopfkontrolle im Vordergrund. Es werden Gesicht und Mund stimuliert und zunächst das Schlucken des Speichels angebahnt. Die Voraussetzungen zur oralen Nahrungsaufnahme werden erarbeitet. Die Konsistenz der Nahrung wird der individuellen Schluck-Fähigkeit des Patienten angepasst. Das Essen wird angereicht; später wird der Patient beim Essen begleitet, bis eine sichere, aspirationsfreie Nahrungsaufnahme möglich ist.

\section{Stimulierende Mundpflege}

Der Gesicht-Mundbereich wird durch taktile, thermale und gustatorische Reize stimuliert. Ziel ist es, willkürliche oro-faziale Bewegungen anzubahnen sowie die Hand-Mundkoordination zu fördern. Der Patient wird angeregt, mundmotorische Aufforderungen auszuführen.

Durch gezieltes Vorgehen wird der Mundbereich spürbarer, die Vigilanz erhöht sich und das Schlucken wird angebahnt.

\section{Essen und Trinken}

Essensbegleitung kann bei neurologischen Patienten aus unterschiedlichen Gründen erforderlich sein, z.B. bei:

Einschränkungen der oralen Sensibilität oder Motorik (Nahrungsreste verbleiben im Mund, eingeschränkter Nahrungstransport),

Facialisparese unterschiedlicher Ausprägung (Mundschluss nicht ausreichend möglich), neuropsychologischen Defiziten, Neglect und anderen Aufmerksamkeitsproblemen (keine Orientierung auf das Essen),

Apraxie (Hantieren mit Besteck erschwert/unmöglich).

Bei Patienten mit beeinträchtigtem Schlucken werden schluckaktfördernde Bewegungsmuster, Kopf- und Körperhaltungen in Absprache mit der logopädischen bzw. ergotherapeutischen Schlucktherapie systematisch eingesetzt, die Konsistenz von Nahrungsmitteln und Flüssigkeiten modifiziert und die Schluckkompetenz durch Einfärben kontrollierbar gemacht.

Patienten mit sicherer Schluckfähigkeit werden ihren Möglichkeiten entsprechend zunehmend in die aktive Nahrungsaufnahme mit einbezogen, z. B. Vorbereitung, Führen der Patientenhand zum Mund, Förderung der Koordination zwischen Hand und Mund, Training des bimanuellen Umgangs mit Besteck, ggf. Training von Hilfsmitteln wie Einhänderbrett.

\section{Kommunikation}

Erkennen und gezieltes Beantworten von Kommunikationssignalen des Patienten verbaler, nonverbaler akustischer, gestischer und mimischer Art (dialogischer Kommunikations- 
aufbau). Bei schwersten Kommunikationsstörungen regelmäßige Wiederholung einfacher Kommunikationssignale in verschiedenen zusammengehörigen Sinneskanälen (taktil, verbal, gestisch, mimisch).

\section{Gedächtnis und Orientierung}

Förderung der Tagesstrukturierung durch tageszeitadäquate Beleuchtung. Verbale Informationen zu Tag, Tageszeit, Ort und zu den Therapien werden regelmäßig wiederholt, insbesondere im Bezug zu typischen Verrichtungen wie morgendliches Waschen und Ankleiden, abendliches Waschen und Entkleiden usw. Tagesstrukturierung durch möglichst wochenweise stabile Termine. Ggf. Hilfsmittel, z. B. Kalender, Uhr.

\section{Blasen-|Darmtätigkeit, Toilettentraining}

Sobald durch nonverbale (Unruhe, Nesteln usw.) oder verbale Signale des Patienten anzunehmen ist, dass der Patient Urinoder Stuhldrang verspürt, wird ihm die Urinflasche angelegt oder das Steckbecken gereicht.

Hier werden die Beckenhebung, das Seitwärtsversetzen und das Drehen des Beckens (Rotationsbewegungen der Bauchmuskulatur) angebahnt und eingeübt.

Soweit Vigilanz, Kreislaufregulation und Wahrnehmungsfähigkeit dies erlauben, werden inkontinente Patienten regelmäßig auf den Toilettenstuhl gesetzt. Verbal und situativ wird der Patient hierauf vorbereitet. Eine Vorbereitung kann auch durch Aktivierung der Darmtätigkeit im Rahmen einer Kolonmassage erfolgen. Der Wechsel von Inkontinenzhosen, Unterlagen usw. erfolgt unter Einbeziehung des Patienten, um ihn über seine Ausscheidungsfunktionen $\mathrm{zu}$ informieren, zu aktivieren und eine Eigenkontrolle anzubahnen.

\section{Atmung}

Förderung der Atemtiefe. Dadurch verbesserte Wahrnehmung basaler Vorgänge und damit kognitive Stimulation. Die Förderung der Atmung erleichtert dem Patienten zudem die Sekretmobilisation, aktiviert zum Abhusten und verbessert durch eine kontinuierlich gute Sauerstoffzufuhr alle Kreislaufparameter sowie Vigilanz und Kognition.

\section{Absaugen/Trachealkanülenmanagement}

Primäres Ziel sind freie geschützte Atemwege. Eine zügige Dekanülierung wird angestrebt. Dies erfolgt über Stimulation des Schluckvorgangs, Anfärben des Speichels, Anpassung einer adäquaten Trachealkanüle, Verlängerung der Entblockungszeiten und Einsatz eines Sprechventils unter kontinuierlicher Überwachung.

\section{Management bei Isolierung infolge Besiedlung} multiresistenter Keime

Aufwändige und zeitintensive Isolierungs-, Eradikations- und Hygienemaßnahmen.

Isolierungsmaßnahmen können den Patienten in seiner Eigenaktivität bei den ATLs einschränken, seine Unsicherheiten und Ängste verstärken und ein Gefühl der Ausgrenzung verursachen. Dieser vermehrten psychischen Belastung wird durch bewusst intensivierte Zuwendung, durch Kontakt- und Kommunikationshilfen in der Pflegetherapie wie auch im Rahmen der täglich stattfindenden Therapien entgegengewirkt.

\section{Leistungskatalog der therapeutischen Pflege und Plausibilitätszeiten \\ $\nabla$}

\section{Training der Körperpflege}

TP 1 Waschtraining komplett [20 min]

- Beruhigende/belebende GKW im Bett zur Einflussnahme auf Vigilanz und Stress

- Wahrnehmungsfördernde GKW im Bett mit verbaler, haptischer und taktiler Unterstützung

- Aktivierende Körperpflege im Sitzbett

- Aktivierende Körperpflege in der Seitenlage

- Aktivierende Körperpflege neben dem Bett

- Aktivierende Körperpflege sitzend am Waschbecken

- Aktivierende Körperpflege stehend am Waschbecken

- Unterstützung bei Wahrnehmungsstörungen (Hemineglect, Hemianopsie) z.B. durch geführtes Waschtraining (Training der Struktur und des zielgerichteten Handelns) (Schulung im Umgang mit bewegungseingeschränkten Extremitäten [Paresen ...])

\section{TP 2 Waschtraining inkomplett [10 min]}

- Waschtraining einzelner Körperabschnitte

- Waschtraining zur Durchführung der Abendtoilette

- Geführtes Waschtraining bei Wahrnehmungsstörungen

TP 3 Duschen oder Baden [20 min]

- Aktivierende Körperpflege beim Duschen/Baden

- Stimulation und Wahrnehmungstraining in der Badewanne (Selbsthilfetraining beim Duschen/Baden)

(Schulung im Umgang mit bewegungseingeschränkten Extremitäten [Paresen ...]) (Unterstützung bei Wahrnehmungsstörungen [Hemineglect, Hemianopsie])

(Schulung im/Hilfe beim Umgang mit geeigneten Hilfsmitteln)

TP 4 Persönliche Pflege [5 min]

- Wahrnehmungstraining auf Grundlage dem Patienten bekannter Handlungen wie Rasur, Haarpflege, Schminken, spezielle Hautpflege

- Selbsthilfetraining in den Handlungen Rasur, Haarpflege, Schminken, spezielle Hautpflege

- Waschtraining von Gesicht und Händen nach Mahlzeiten (Schulung im Einsatz geeigneter Hilfsmittel)

\section{TP 5 Stimulierende Mundpflege [5 min]}

- Stimulation durch taktile, thermische oder gustatorische Reize, siehe Beschreibung im Konzept unter „Stimulierende Mundpflege“

TP 6 Mundpflege [5 min]

- Stimulation und Anbahnung alltagsrelevanter Bewegungen bei der Mundpflege

- Selbsthilfetraining zur Mundpflege nach den Mahlzeiten (Schulung im/Hilfe beim Umgang mit geeigneten Hilfsmitteln)

\section{TP 7 An-|Ausziehtraining [10 min]}

- Wahrnehmungsschulung beim Kleiderwechsel inkl. Schuhe und ATS mit verbaler, haptischer und taktiler Unterstützung unter Verwendung patienteneigener Kleidung und Schuhe

- Aktivierung und Anbahnung alltagsrelevanter Bewegung beim An-/Ausziehen 
- Selbsthilfetraining beim kompletten Kleiderwechsel inkl. Schuhe und ATS

(Unterstützung bei Wahrnehmungsstörungen [Hemineglect, Hemianopsie])

(Schulung im/Hilfe beim Umgang mit geeigneten Hilfsmitteln)

(Schulung im Umgang mit bewegungseingeschränkten Extremitäten [Paresen ...])

TP 8 Kontinenztraining [5 min]

- Einüben der Beckenhebung

- Erlernen/Fördern der Bauchpresse

- Ggf. Crede-Handgriff oder Blasentriggern

- Kolonmassage

(Patient regelmäßig an die Blasen- und Darmentleerung erinnern und die Urinflasche anlegen oder das Steckbecken reichen)

\section{TP 9 Toilettentraining [10 min]}

- Regelmäßige Toilettengänge (WC oder Nachtstuhl) durchführen und die Entleerung anbahnen und unterstützen (Schulung im/Hilfe beim Umgang mit geeigneten Hilfsmitteln und Inkontinenzartikeln)

(Einüben der normalen Abläufe, die zur Ausscheidung auf der Toilette erforderlich sind [An-/Auskleiden])

(Schulung im/Hilfe beim Führen eines Miktionsprotokolls) (Umgebung sicher gestalten, Beaufsichtigung bei Sturzgefahr)

\section{Ernährung}

TP 10 Esstraining bei Kau-/Schluckstörung [20 min]

- Esstraining bei Kau-/Schluckstörung

(Anbahnung und Aktivierung der zur Aufnahme von Speisen und Getränken benötigten Bewegungen)

(Unterstützung des Schluckaktes mittels Kieferkontrollgriff) (Speisen und Getränke adaptieren)

(Für eine geeignete Atmosphäre/Umgebung sorgen)

(Arbeitstempo reduzieren bzw. zum langsamen Essen [Kauen] anhalten) (wiederholt verbal wie taktil Aufmerksamkeit auf das Essen lenken)

(Hilfe beim Umgang mit geeignetem Besteck/Geschirr; verbale/olfaktorische Unterstützung)

(Stimulation/Einübung des Schluckaktes/Inspektion der Mundhöhle)

TP 11 Esstraining ohne Kau-/Schluckstörung [15 min]

- Esstraining ohne Kau-/Schluckstörung

(Anbahnung und Aktivierung der zur Aufnahme von Speisen und Getränken benötigten Bewegungen)

(Eingehen auf die Wahrnehmungsstörung des Patienten im Tempo und Ablauf)

(Für eine geeignete Atmosphäre/Umgebung sorgen)

(Patient in das Richten der Speisen/Getränke mit einbeziehen)

(Schulung im/Hilfe beim Umgang mit geeigneten Hilfsmitteln/ Prothese, Einhänderbrett, Griffverdickung, Becher, rutschfeste Unterlagen, Strohhalm, Spiegel usw.)

(Hände mit geeignetem Besteck/Geschirr führen)

(Training des bimanuellen Führens von Besteck)

(Verbale/olfaktorische Unterstützung)

(Anschließende Mundpflege und Inspektion der Mundhöhle)

(Vorlieben/Unverträglichkeiten erfragen und einbeziehen)

(Soziale Integration des Patienten beim Essen)

\section{Therapeutische Positionierung (Lagerung) und} Mobilität

TP 12 Mobilisation [10 min]

- Anbahnung und Aktivierung alltagsrelevanter Bewegungen zum Lagewechsel, Schulung im Umgang mit bewegungseingeschränkten Extremitäten

- Training der Bauch- und Beckenmuskulatur, Rotationsbewegungen, Bridging, Becken versetzen

- Zum Kopfende bewegen nach neurophysiologischen Prinzipien (z.B. Bobath oder kinästhetisch)

- Ggf. Einüben von Kompensationsstrategien (Bettgitter nutzen)

- Mobilisation vom Liegen in den Bettkantensitz trainieren, Stützfunktion Arme/Beine

- Erarbeiten von statischer/dynamischer Sitzstabilität, Bettkantensitz, Gleichgewichtstraining im Sitzen

- Anleitung/Begleitung physiologischer Alltagsbewegungen mit verbaler Unterstützung

TP 13 Therapeutische Positionierung (Lagerung) [10 min]

- Positionswechsel unter Einsatz therapeutischer aktivierender Lagerungstechniken

- Atemunterstützende Lagerungen, V; A; T; I-Lagerungen

- Wahrnehmungsfördernde Nestlagerung

- Seitenlagerung in 30, 60, 90 oder $120 \mathrm{Grad}$

- Übernahme geeigneter Lagerung zur Aufnahme von Speisen/ Getränken

- Erarbeiten einer geeigneten Ausgangsposition zur Aufnahme von Speisen und Getränken

- Positionierung in Neutral-Stellung, Bauchlagerung, Sitzbett (Lagerungen sinnvoll in Tagesablauf integrieren [Therapieplan])

TP 14 Therapeutischer Transfer [5 min]

- Alle Handlungsteilschritte, die zur Vorbereitung, Ausführung und Beendigung des Transfers nötig sind, einüben

- Übernahme des Transfers vom Bett in Rollstuhl oder zurück mittels tiefen Transfers

- Transfer über den Stand

- Kinästhetischer Transfer (Schulung im Umgang mit bewegungseingeschränkten Extremitäten [Paresen ...])

(Unterstützung bei Wahrnehmungsstörungen [Hemineglect, Hemianopsie])

(Schulung im/Hilfe beim Umgang mit geeigneten Hilfsmitteln)

\section{TP 15 Sturzprophylaxe [5 min]}

- Umgebung des Patienten sicher gestalten, um Stürze zu vermeiden und Sturzfolgen auf ein Minimum zu reduzieren (freier Weg zur Toilette, keine Stolperfallen auf dem Boden, für erreichbare Klingel sorgen, nicht zu lange Kleidung anziehen, welche auf dem Boden schleift, schlecht sitzende Schuhe können zu Gehunsicherheiten führen, Brillenglasstärken bei Bedarf anpassen lassen, Hüftprotektoren anbieten)

- Begleitung des Patienten beim Kraft- und Balancetraining mit dem Ziel, ein Stolpern abzufangen

- Eventuelle Sturzgefahren erkennen und vermeiden

- Auf Haltegriffe in Bad, Toilette und Flur hinweisen und deren Benutzung gezielt beüben

- Einrichten eines geschützten Bettenplatzes, z.B. Bodenlagerung im Patientenzimmer oder Einsatz eines Sturzbettes 
- Beratung und Schulung von Patienten und Angehörigen zur Sturzprophylaxe. Wenn möglich, Maßnahmen gemeinsam planen und bis zur Evaluation begleiten

TP 16 Gehübungen [5 min]

- Gehübungen unter verbaler und taktiler Korrektur des Gangbildes

- Schulung im Umgang mit bewegungseingeschränkten Extremitäten (Paresen ...)

- Unterstützung bei Wahrnehmungsstörungen (Hemineglect, Hemianopsie)

- Schulung im/Hilfe beim Umgang mit geeigneten Hilfsmitteln (Umfeld sicher und sinnvoll gestalten [freier Weg zur Toilette, keine Stolperfallen auf dem Boden])

(Übungen sinnvoll in Tagesablauf integrieren [Therapieplan, Toilettengang, Mahlzeiten])

\section{TP 17 Stehübungen [10 min]}

- Anbahnung und Einüben des Aufstehens

- Schulung im Umgang mit bewegungseingeschränkten Extremitäten (Paresen ...)

- Unterstützung bei Wahrnehmungsstörungen (Hemineglect, Hemianopsie)

- Stehbett

- Standing

- Stehen im Life-Stand

- Schulung im/Hilfe beim Umgang mit geeigneten Hilfsmitteln (Übungen sinnvoll in Tagesablauf integrieren)

TP 18 Bewegungstrainer [10 min]

- Bewegungstrainer für untere und obere Extremitäten einsetzen. Die Anwesenheit des Pflegetherapeuten während der Therapie ist wichtig. Dabei wird u.a. auf Änderungen im Tonus geachtet. Währenddessen wird unterstützend reagiert, indem die Sitzposition kontinuierlich angepasst wird.

\section{Kognitive und emotionale Aktivitäten}

TP 19 Orientierungstraining [5 min]

- Fortführen und Unterstützen des kognitiven Trainings im Stationsalltag durch gezieltes Üben zur Orientierung auf Station/ Klinikebene (eigener Patientenschrank, Aufenthaltsräume, Toiletten), Nutzen von Schildern oder Piktogrammen

- Biografiearbeit (wenn möglich, mit Bildmaterial von Angehörigen)

- Einbezug des Kalenders, Uhren

- Gemeinsames Erarbeiten eines Tagesplanes (schriftlich)

(Pflegetherapeutisches Begleiten im Tagesverlauf bei sämtlichen ADL-Handlungen [z.B. WAT, Essensbegleitung])

(Begleitend zu allen Maßnahmen bewusstes Hinweisen auf Räumlichkeiten, Tageszeit, Handlungen durch betreuende Personen)

(Bei jedem Patientenkontakt deutliches Vorstellen der eigenen Person)

(Auf personelle und inhaltliche Kontinuität durch Bezugspersonen bei Pflegetherapie achten)

(Tag-/Nacht-Rhythmus gewährleisten, Tagesablauf strukturieren)

(Patienteneigene Kleidung und Pflegemittel nutzen)

(Individuelle Zimmer- und Bettplatzgestaltung mit persönlichen Gegenständen, Farben, Symbolen)
TP 20 Gedächtnistraining [5 min]

- Gedächtnistagebuch führen

- Gezieltes Informieren über Zeit, Ort und Situation und Abfragen einzelner Inhalte

(Tag-/Nacht-Rhythmus gewährleisten, Tagesablauf strukturieren)

(Auf personelle und inhaltliche Kontinuität bei Pflegetherapie achten)

TP 21 Situative Krisenintervention [5 min]

- Komplexleistung Situative Krisenintervention

(Aktives Zuhören/Validation)

(Für passendes Umfeld sorgen)

(Gewährung von ausreichend Zeit, um Gefühle äußern zu können) (Multiprofessionelle Beratung)

(Zeit für Bewältigung lassen) (Flexibilität in Pflege und Therapie ermöglichen)

(Ärztliche/Psychologische Hilfe einleiten)

\section{TP 22 Motivation und Antrieb [10 min]}

- Gemeinsames Erarbeiten und Überprüfen von Zielen in Zusammenarbeit mit Angehörigen und/oder therapeutischem Team

- Zwischenschritte zur Erreichung des Rehabilitationsziels aufzeigen, gemeinsam mit dem Patienten planen

- Anreize setzen und Erfolge aufzeigen

TP 23 Affektregulation [5 min]

- Komplexleistung Affektregulation

(Fester Bezugsrahmen) (Strukturierungshilfen zur Alltagsbewältigung durch die Pflege)

(Aktives Zuhören/Validation)

(Verbalisieren)

(Für passendes Umfeld sorgen)

(Multiprofessionelle Beratung)

\section{TP 24 Absaugen [5 min] \\ - Absaugen}

TP 25 Versorgung von Patienten mit Trachealkanüle/-Entwöhnung [15 min]

- Komplexleistung Versorgung von Patienten mit Trachealkanüle/-Entwöhnung

(Reinigung/Verbinden des Tracheostomas)

(O2-Therapie vor Absaugen und Entblocken)

(Cuffdruck-Kontrolle bei geblockter Kanüle)

(Reinigung und Wechsel von Inlay [Innenkanüle])

(Ggf. Einsetzen des Sprechventils)

(Ggf. Speichel einfärben)

(Schluck-, Atem- und Hustentraining)

TP 26 Trachealkanülenwechsel [30 min]

- Wechsel der Trachealkanüle inkl. Vorbereitung, Verbandwechsel und Nachsorge durch 2 Fachkräfte

TP 27 Management bei Isolierung infolge Besiedlung durch multiresistente Keime [ 60 min, maximal einmal je Tag]

- Maßnahmen nach hauseigenen Standards und Hygienerichtlinien einhalten

(Schulung/Information der Angehörigen über Verhaltensregeln) 


\section{TP 28 Wahrnehmungstraining [5 min]}

- Individuelle Förderangebote durch das Setzen von akustischen, sensorischen, olfaktorischen und optischen Reizen. Dem Patienten Zeit für eine Reaktion lassen

- Wahrnehmungsförderung durch Ausstreichen von Körperregionen/Extremitäten/Körperkonturen

- ASE (Atemstimulierende Einreibungen)

\section{TP 29 Kommunikationstraining [5 min]}

- Nonverbale Kommunikation etablieren und dokumentieren: Blinzeln, Seufzen, Händedruck ... Erarbeiten des Signalisierens von Zustimmung und Ablehnung

- Externe Steuerung (Ansprache, Berührung, Führen) der Aufmerksamkeitsausrichtung im Handlungsrahmen

- Externe Steuerung der Aufmerksamkeit auf den nächsten Handlungsschritt (externe Strukturierung)

- Steuerung der Aufmerksamkeit auf die sprachlichen Äußerungen (durch Akzentuierung, Wiederholung usw.) eingebettet in nonverbale Handlungen

- Training des Verstehens sprachlicher Äußerungen eingebettet in nonverbale Handlungen (parallel)

- Training von Konversationsroutinen (Kontaktaufnahme, Adressierung, minimale Kooperation: Sprecherwechsel)

- Schulung im/Hilfe beim Umgang mit geeigneten Hilfsmitteln; Buchstabentafeln, Piktogramme einsetzen

- Wichtige Themen (mit den Angehörigen) zu verschiedenen Zeitpunkten mehrmals besprechen

(Ansprache und verbale Begleitung bei Pflegetherapiemaßnahmen)

(Sensibles Korrigieren und Trainieren bei Aphasien, Stoppen bei Jargon und Automatismen)

(Interpretation der Äußerungsversuche des Patienten: immer wieder absichern)
(Verständigungssicherung: die Bedeutung der sprachlichen Äußerung und Konsequenz des Nichtverstehens erfahrbar machen)

(Rückfragen, bestätigen lassen)

(Gegebenenfalls Zeigen, Zeichnen, eindeutige Gesten, Stichworte schriftlich fixieren)

TP 30 Angehörigenberatung/-schulung,

Pflegeüberleitung [15 min]

- Gezielte, strukturierte und geplante Gespräche mit Angehörigen

- Angehörigen-Hospitationen auf Station organisieren und nach Wunsch Angehörige gezielt anleiten

- Einweisung in Rooming-in

- Organisieren, Schulen und Anleiten der Angehörigen im Hilfsmittelgebrauch

- Kontaktaufnahme mit externen Pflegediensten und anderen Kooperationspartnern, um diese bei Bedarf in Hilfsmittel einzuweisen

\section{TP 31 Dokumentationszeiten. Zeitaufschlag pro Tag}

[15 min]

- Die schriftliche Dokumentation der pflegetherapeutischen Leistungen dient der Qualitätssicherung bei der Versorgung neurologischer Frührehabilitationspatienten.

- In der Minutenpauschale wird die Dokumentation der allgemeinen und speziellen Pflege nicht berücksichtigt.

- Der pflegerische Zeitaufwand für die wöchentliche ICF-Besprechung und deren Dokumentation fließen nicht in die Minutenabrechnung mit ein, sondern sind durch die Pauschale ebenfalls abgedeckt. 